\title{
Real-time 3-D hybrid simulation of Titan's plasma interaction during a solar wind excursion
}

\author{
S. Simon \\ Institute of Geophysics and Meteorology, University of Cologne, Germany \\ Institute for Theoretical Physics, TU Braunschweig, Germany \\ Received: 1 April 2009 - Revised: 27 July 2009 - Accepted: 19 August 2009 - Published: 1 September 2009
}

\begin{abstract}
The plasma environment of Saturn's largest satellite Titan is known to be highly variable. Since Titan's orbit is located within the outer magnetosphere of Saturn, the moon can leave the region dominated by the magnetic field of its parent body in times of high solar wind dynamic pressure and interact with the thermalized magnetosheath plasma or even with the unshocked solar wind. By applying a threedimensional hybrid simulation code (kinetic description of ions, fluid electrons), we study in real-time the transition that Titan's plasma environment undergoes when the moon leaves Saturn's magnetosphere and enters the supermagnetosonic solar wind. In the simulation, the transition between both plasma regimes is mimicked by a reversal of the magnetic field direction as well as a change in the composition and temperature of the impinging plasma flow. When the satellite enters the solar wind, the magnetic draping pattern in its vicinity is reconfigured due to reconnection, with the characteristic time scale of this process being determined by the convection of the field lines in the undisturbed plasma flow at the flanks of the interaction region. The build-up of a bow shock ahead of Titan takes place on a typical time scale of a few minutes as well. We also analyze the erosion of the newly formed shock front upstream of Titan that commences when the moon re-enters the submagnetosonic plasma regime of Saturn's magnetosphere. Although the model presented here is far from governing the full complexity of Titan's plasma interaction during a solar wind excursion, the simulation provides important insights into general plasma-physical processes associated with such a disruptive change of the upstream flow conditions.
\end{abstract}

Keywords. Magnetospheric physics (Magnetosphere interactions with satellites and rings; Magnetosphere-ionosphere interactions) - Space plasma physics (Kinetic and MHD theory)

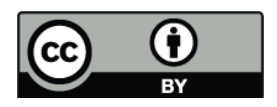

Correspondence to: S. Simon (sven.simon@tu-bs.de)

\section{Introduction}

Since the arrival of the Cassini spacecraft at Saturn in July 2004, newly collected plasma and magnetic field data have greatly improved our knowledge of the properties of Saturn's numerous moons. Of particular interest is the interaction between Saturn's moons and the corotating plasma of the magnetosphere, into which most of the giant planet's satellites are embedded. Because the Keplerian speeds of the moons are considerably smaller than the velocity of the at least partially corotating magnetospheric plasma, the moons are continuously overtaken by the magnetospheric flow. The global morphology of the resulting plasma interaction process does not only depend on the characteristics of the impinging plasma flow (density, temperature, Mach numbers etc.), but the features of the moon itself also play a decisive role. In general, the electromagnetically inert icy satellites leave a significantly weaker imprint on the plasma flow pattern and the magnetic field topology than an obstacle that possesses a dense ionosphere.

Special interest has been devoted to the plasma interaction of Titan, because - except for Earth - this moon is the only body in the solar system that possesses a dense, nitrogenrich atmosphere. Saturn's magnetic field lines strongly drape around Titan's ionosphere, while newly generated exospheric ions are being picked up and incorporated into the incident plasma. The gyroradii of these particles can exceed the diameter of Titan by about an entire order of magnitude (Luhmann, 1996). Therefore, effects that are associated with the finite ion gyroradius play an important role for the overall topology of Titan's plasma environment. Specifically, the exospheric pick-up tail as well as the magnetic pile-up region at Titan's ramside exhibit a pronounced asymmetry with respect to the direction of the convective electric field in the impinging magnetospheric plasma (Brecht et al., 2000; Simon et al., 2006b, 2007b; Modolo and Chanteur, 2008).

So far, Cassini has accomplished more than 50 flybys of Titan. Magnetic field observations during the first series of

Published by Copernicus Publications on behalf of the European Geosciences Union. 
encounters in 2004 and 2005 have been interpreted by Ma et al. (2004, 2006) as well as Backes et al. (2005), Backes (2005) and Neubauer et al. (2006), whose models are based upon a magnetohydrodynamic description of the plasma flow around Titan. Special interest has been devoted to the Cassini T9 flyby of Titan which took place in December 2005. Since during T9, the spacecraft passed through Titan's induced magnetotail at a distance of about $4 R_{T}$ (radius of Titan: $R_{T}=2575 \mathrm{~km}$ ) to the moon, this encounter offered a unique chance to study the magnetic lobe structure in Titan's wake region. Several global numerical models have been applied to investigate the influence of the upstream plasma composition, the direction of the impinging magnetospheric flow and the total ion production rate in the moon's ionosphere on the structure of Titan's induced magnetotail during T9 (Kallio et al., 2007; Ma et al., 2007; Modolo et al., 2007; Simon et al., 2007c). These studies come to the conclusion that during $\mathrm{T} 9$, the bulk speed of the impinging magnetospheric plasma was not aligned with the direction of ideal corotation, but the flow was directed away from Saturn in an angle of about $30^{\circ}$. Magnetic field observations during T34, a recent passage through Titan's ramside ionosphere that took place at about 18:00 Saturnian local time, have been quantitatively reproduced by Simon et al. (2008) who applied a hybrid simulation code.

All simulation models discussed above assume the parameters of the impinging magnetospheric plasma to be constant in space and time. However, for the plasma environment of Titan, this assumption is not always appropriate. Titan's orbit is located within the outer regions of Saturn's magnetosphere at a distance of about 20 Saturn radii. Therefore, when Saturn's magnetosphere is compressed in times of high solar wind dynamic pressure, Titan can leave the magnetosphere of its parent body near noon local time and interact with the shocked solar wind plasma in Saturn's magnetosheath or even directly with the unshocked solar wind. During the T32 encounter on 13 June 2007, Cassini became the first spacecraft to provide in-situ observations of Titan's plasma environment outside the magnetosphere of Saturn, as the moon was located within the shocked solar wind plasma of the magnetosheath at the time of closest approach. Until only a few minutes before the encounter, Titan had been exposed to the corotating plasma inside Saturn's magnetosphere (Bertucci et al., 2008). During the flyby, the ambient magnetic field nearly reversed its direction. The density and composition of the impinging plasma flow also changed dramatically during the pass (Coates et al., 2007). Within the framework of a hybrid model, Simon et al. (2008, 2009a) recently studied how Titan's plasma environment is affected by such sudden changes in the upstream plasma and magnetic field conditions. These authors presented the first global, self-consistent model of Titan's plasma interaction that allows to include non-stationary upstream conditions. In their studies of the T32 scenario, reversals of the external magnetic field direction as well as changes in the density and composition of the impinging plasma flow are considered. It is demonstrated that when Titan crosses Saturn's magnetopause boundary, the satellite's induced magnetosphere is reconfigured due to reconnection, with the time scale of the reconfiguration process being determined by the convection of the magnetic field lines in the undisturbed, impinging plasma flow. In consistency with the interpretation of Cassini magnetometer data from the T32 encounter (Bertucci et al., 2008), the picture drawn by the hybrid model suggests that Titan opened Saturn's magnetopause like a comet that crosses a sector boundary in the solar wind. Moreover, Simon et al. $(2008,2009$ a) show that the reconfiguration of Titan's exospheric tail during the magnetopause passage is controlled by the large gyroradii of the involved pick-up species, but the change in the upstream plasma conditions does not lead to a detachment of the tail. It should be noted that while this model considered both a change of the plasma composition (from oxygen to hydrogen) and a magnetic field reversal at the boundary layer between both plasma regimes, the magnetospheric as well as the magnetosheath flow possessed a magnetosonic Mach number that is smaller than $M_{S}=1$.

Even though no in-situ data are currently available for this situation, Bertucci et al. (2008) also suggest that a few hours before the T32 encounter, Titan may have been exposed to the unshocked, superfast solar wind upstream of Saturn's bow shock. The authors come to this conclusion by applying an empirical model of Saturn's bow shock developed by Masters et al. (2008). In such a scenario, Titan's plasma environment is expected to undergo an even more drastic transition than during a magnetosheath excursion. When being located in the solar wind, a bow shock should arise in front of Titan, and the deflection of the rather cold solar wind around the obstacle should be significantly stronger than the imprint that Titan leaves on the hot plasma flow in Saturn's outer magnetosphere. When being located in the solar wind, Titan's plasma environment should exhibit the same structures as the induced magnetospheres of Mars and Venus. Especially, a bow shock and an Ion Composition Boundary should be formed (cf. e.g. Sauer et al., 1994; Simon et al., 2007a, for details).

Although the interaction between Titan and a supermagnetosonic solar wind flow has already been studied within the framework of several fluid and hybrid plasma models (Kallio et al., 2004; Ledvina et al., 2004; Simon et al., 2006b; Simon, 2007), the transition that Titan's plasma environment undergoes when the moon leaves Saturn's magnetosphere and enters the supermagnetosonic solar wind has so far not been studied in a real-time simulation scenario. Within the framework of this work, we therefore present the first application of a global numerical real-time model to Titan's plasma interaction during an excursion from the magnetosphere to the superfast solar wind. Our hybrid simulation model is a modified version of the code that has recently been successfully applied to the T32 magnetosheath excursion (Simon et al., 2009a). The purpose of our study is not only to investigate 
the transition that Titan's induced magnetosphere undergoes when the moon enters the solar wind, but we also analyze in real-time the removal of the newly formed shock front ahead of Titan that commences when the moon re-enters Saturn's magnetosphere. Based on the simulation results, we try to determine the characteristic time scales on which Titan's induced magnetosphere is reconfigured.

This article is organized as follows: in Sect. 2, we provide a description of our hybrid simulation code and give an overview of the set of input parameters. Since the model itself has been discussed in detail in our companion publications cited above, we do not dwell into details, but only a brief overview is given. Sections 3 and 4 deal with the results of our real-time simulations. In Sect. 3, the case of Titan leaving Saturn's magnetosphere and entering the superfast solar wind is discussed. In Sect. 4, Titan's re-entry into the submagnetosonic magnetospheric plasma regime is investigated. The paper concludes with a summary of our major findings (Sect. 5).

\section{Model description and input parameters}

The hybrid model treats the electrons of the plasma as a massless, charge-neutralizing fluid, whereas the ions are represented by individual particles. Therefore, the major strength of this simulation approach is the capability to resolve effects that arise from the finite ion gyroradius, such as velocity differences between upstream and pick-up species or non-Maxwellian particle distribution functions. In recent years, the hybrid model upon which the present study is based has been employed to analyze various aspects of Titan's plasma interaction (cf. our companion papers listed above). Besides, the model has been successfully applied to the interaction between the Martian ionosphere and the solar wind (Simon et al., 2007a; Bößwetter et al., 2007), to cometary plasma interactions (Bagdonat and Motschmann, 2002; Motschmann and Kührt, 2006) and to the plasma environment of weakly magnetized asteroids (Simon et al., 2006a). Within the framework of the Cassini mission, the very weak magnetic field perturbations observed in the wakes of Saturn's icy satellites Tethys and Rhea have been successfully reproduced as well (Roussos et al., 2008; Simon et al., 2009b). Recently, a first application of our hybrid code to the plasma interaction of Enceladus has been presented at the AGU Fall Meeting (Kriegel et al., 2008; Kriegel, 2009). The authors conducted a systematic analysis of how particle absorption at the surface of Enceladus and pick-up of ions from the plume below its south pole contribute to the overall structure of the interaction region in isolation and collectively. Besides, they succeeded in reproducing Cassini magnetic field observations from the first series of Enceladus flybys in 2005 (Dougherty et al., 2006).

\subsection{Hybrid equations and simulation setup}

Since a detailed description of our model can be found in the preceding publications cited above, only a brief overview of the basic equations shall be given here:

- Equations of motion for individual ions:

$$
\frac{\mathrm{d} \underline{x}_{s}}{\mathrm{~d} t}=\underline{v}_{s} \quad \text { and } \quad \frac{\mathrm{d} \underline{v}_{s}}{\mathrm{~d} t}=\frac{q_{s}}{m_{s}}\left\{\underline{E}+\underline{v}_{s} \times \underline{B}\right\},
$$

where $\underline{x}_{S}$ and $\underline{v}_{s}$ denote the position and the velocity of an ion of species $s$, respectively. The vectors $\underline{E}$ and $\underline{B}$ are the electromagnetic fields. The ion mass and charge are denoted by $m_{s}$ and $q_{s}$, respectively.

- Electric field equation:

$$
\underline{E}=-\underline{u}_{i} \times \underline{B}+\frac{(\nabla \times \underline{B}) \times \underline{B}}{\mu_{0} e n_{e}}-\frac{\nabla P_{e, 1}+\nabla P_{e, 2}}{e n_{e}}
$$

where $\underline{u}_{i}$ is the mean ion velocity. The plasma is quasi-neutral, i.e. the mean ion $\left(n_{i}\right)$ and electron density $\left(n_{e}\right)$ are assumed to be equal. As in general, the electron temperature in a planetary ionosphere differs significantly from the electron temperature in the ambient plasma flow, two different electron pressure terms $P_{e, 1}$ and $P_{e, 2}$ have been incorporated into the simulation model. Specifically, a two-species/single-fluid description is applied to the electrons, i.e. the model discriminates between the densities of the two electron populations, but both of them are assigned the same velocity. In our Titan simulations, the first pressure term refers to the ambient magnetospheric (or solar wind) flow, while the second one represents Titan's ionospheric electrons. Details are discussed in our companion papers (cf. especially Eqs. 4-9 in Simon et al., 2006b, and Eqs. 2-8 in Simon et al., 2007b). Both electron populations are described by adiabatic laws:

$$
P_{e, 1} \propto \beta_{e, 1} n_{e, 1}^{\kappa} \quad \text { and } \quad P_{e, 2} \propto \beta_{e, 2} n_{e, 2}^{\kappa},
$$

where $\beta$ are the plasma betas and $\kappa$ is the adiabatic exponent. For the simulations presented in this work, a value of $\kappa=2$ has been chosen (Bößwetter et al., 2004; Simon et al., 2006a,b).

- Magnetic field equation: By using Faraday's law, an expression describing the time evolution of the magnetic field can be obtained:

$$
\frac{\partial \underline{B}}{\partial t}=\nabla \times\left(\underline{u}_{i} \times \underline{B}\right)-\nabla \times\left[\frac{(\nabla \times \underline{B}) \times \underline{B}}{\mu_{0} e n_{e}}\right] .
$$

Because of the adiabatic description of the electrons, the electron pressure terms do not appear in this equation (Simon et al., 2007a, 2008). 


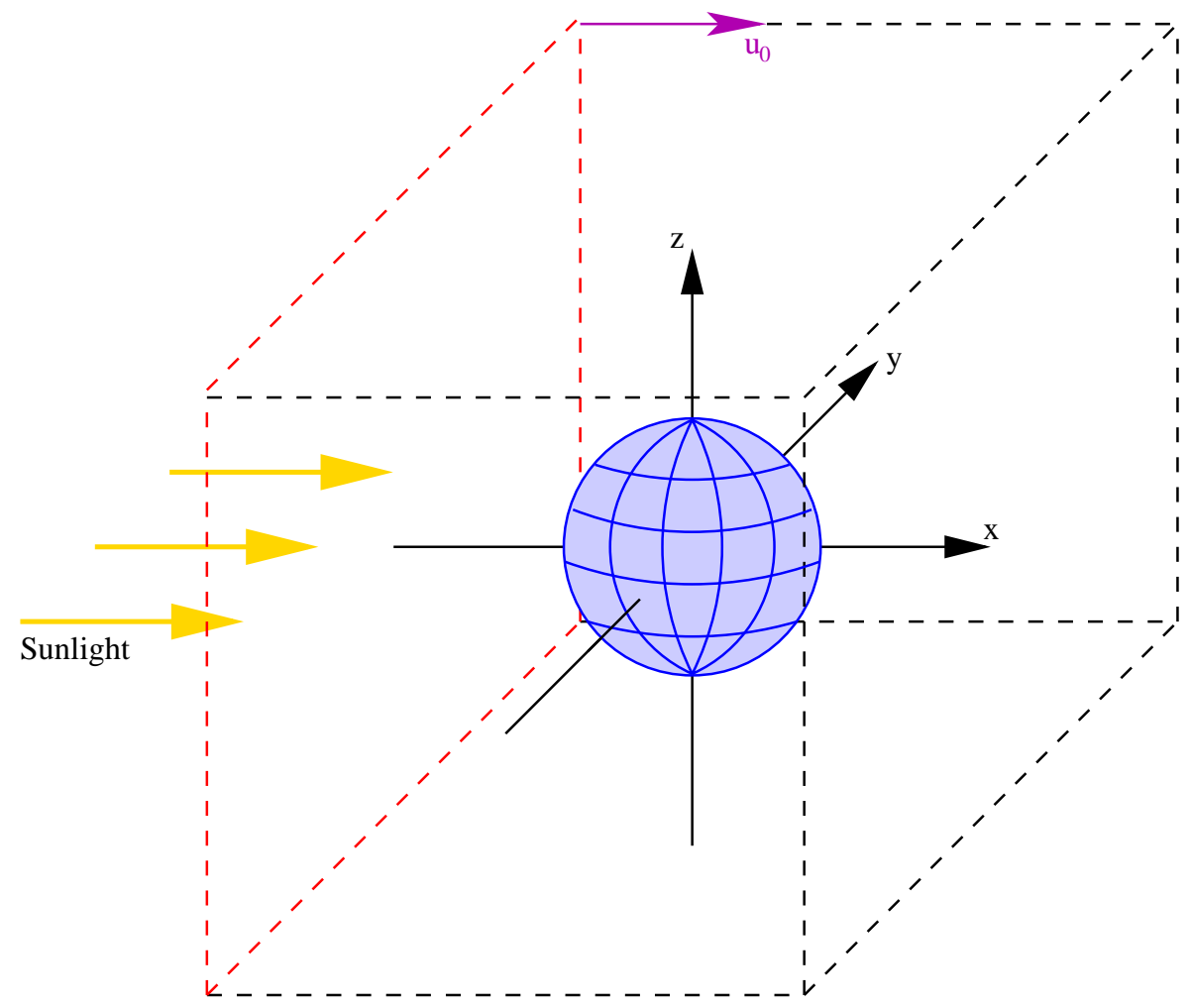

Fig. 1. Simulation geometry. The center of Titan coincides with the center of the cubic simulation box. During the entire simulation, the incident plasma flow is directed along the $(+x)$ axis. The transition from magnetospheric to solar wind plasma regime as well as the subsequent re-entry into the magnetosphere are realized by a modification of the boundary conditions at the left-hand side of the simulation domain (denoted in red). Inside the magnetosphere of Saturn, the undisturbed magnetic field is directed along the $(-z)$ axis. At the boundary to the supermagnetosonic plasma regime, the field direction is reversed.

The simulation was carried out within a cubic domain, featuring a spatial extension of $\pm 7.5 R_{T}$ in each direction (cf. Fig. 1). The center of Titan coincides with the origin of the Cartesian coordinate frame, whose $(x, y)$ plane is identical to the moon's equatorial plane. The positive $z$-axis is antiparallel to the undisturbed magnetic field of Saturn. During the entire simulation, the bulk velocity $\underline{u}_{0}$ of the impinging plasma flow is aligned with the positive $x$ axis. The simulations have been performed on a curvilinear Fisheye Grid, which is adapted to the spherical geometry of the obstacle. This grid consists of $90 \times 90 \times 90$ nodes, i.e. the spatial resolution is of the order of $0.15 R_{T}$. Outflow boundary conditions have been applied to all faces of the simulation domain except for the left wall at $x=-7.5 R_{T}$. At this side of the box, plasma is continuously injected into the simulation domain according to the upstream parameters listed in the subsequent section. Any particle hitting the obstacle boundary at an altitude of $500 \mathrm{~km}$ above Titan's surface is removed from the simulation, whereas the implemented inner boundary conditions allow a free diffusion of the electromagnetic fields through Titan's interior. Details are discussed by Simon et al. (2006b) and especially Simon et al. (2009a).
As implied by the yellow arrows in Fig. 1, Titan's dayside ionosphere is located in the $(x<0)$ half space throughout the entire duration of the simulation. The dayside ion production profile is represented by a Chapman layer, i.e. the local ion production rate depends on the altitude above the surface as well as the solar zenith angle. Ion production due to particle impact at the nightside of Titan (i.e. in the $(x>0)$ hemisphere $)$ is modeled in a phenomenological way, with the production rate depending only on the altitude above the surface, but not on the solar zenith angle. Three ionospheric species of representative masses are considered by our model: molecular nitrogen $\left(\mathrm{N}_{2}^{+}\right)$, methane $\left(\mathrm{CH}_{4}^{+}\right)$and molecular hydrogen $\left(\mathrm{H}_{2}^{+}\right)$. For further details and the specific set of ionospheric input parameters, we would like to refer the reader to our preceding studies (cf. Simon et al., 2006b, 2007b, and especially Sect. 2 in Simon et al., 2009a).

\subsection{Description of real-time simulation approach}

As illustrated in Fig. 2, the simulation scenario applied in this study consists of three steps. During the first step (A) that lasts from $t=0$ until $t=t_{1}=1085 \mathrm{~s}$, Titan is assumed to be located within the corotating plasma of Saturn's 


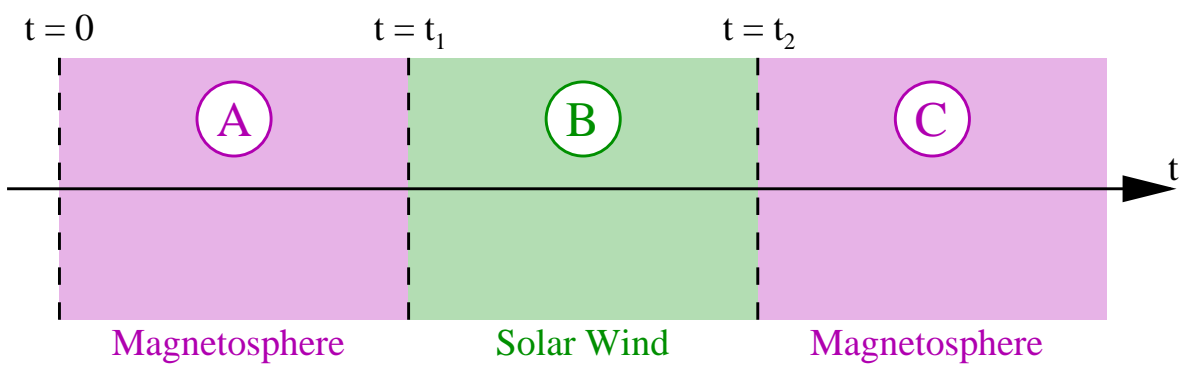

Fig. 2. Schematic illustration of the real-time simulation scenario. In the first part (A) of the simulation, the inflow boundary conditions applied to the left-hand face of the simulation box correspond to the situation inside Saturn's magnetosphere. The plasma entering the box mainly consists of a submagnetosonic flow of oxygen ions. After a quasi-stationary state has been achieved, the boundary conditions are suddenly changed at $t=t_{1}$. In part B of the simulation, the magnetic field that enters the simulation box from the left-hand side is aligned with the $(+z)$ axis, i.e. its direction is reversed with respect to the situation inside the magnetosphere. The plasma entering the box in this part of the simulation consists of a cold, superfast hydrogen population. At $t=t_{2}=2 t_{1}$, the final part (C) of the real-time simulation is initiated. For $t \geq t_{2}$, the boundary conditions at the left-hand side of the simulation domain are the same as in part A and correspond to the situation inside the magnetosphere of Saturn.

magnetosphere. In this situation, the parameters of the magnetospheric plasma entering the simulation box from the left-hand side have been chosen close to the Voyager 1 scenario suggested by Neubauer et al. (1984): The incident plasma flow consists of two ion species, namely oxygen $\left(\mathrm{O}^{+}\right)$and hydrogen $\left(\mathrm{H}^{+}\right)$, their densities being set to $n\left(\mathrm{O}^{+}\right)=0.2 \times 10^{6} \mathrm{~m}^{-3}$ and $n\left(\mathrm{H}^{+}\right)=0.1 \times 10^{6} \mathrm{~m}^{-3}$. The thermal velocities of both ion species,

$v_{t h}=\sqrt{\frac{3 k T_{s}}{m_{s}}}$

( $k T_{s}:$ temperature), have been set to the same value of $v_{t h}=180 \mathrm{~km} / \mathrm{s}$, i.e. their temperatures are $k T\left(\mathrm{O}^{+}\right)=1578 \mathrm{eV}$ and $k T\left(\mathrm{H}^{+}\right)=113 \mathrm{eV}$. The ambient magnetic field is given by $\underline{B}_{0}=(0,0,-5) \mathrm{nT}$. This yields values of $\beta\left(\mathrm{O}^{+}\right)=5.1$ and $\beta\left(\mathrm{H}^{+}\right)=\beta\left(\mathrm{O}^{+}\right) / 28=0.18$ for the plasma betas in the ambient magnetospheric flow. Given an upstream plasma velocity of $\underline{u}_{0}=(120,0,0) \mathrm{km} / \mathrm{s}$, the magnetospheric flow impinging on Titan is superalfvénic (alfvénic Mach number: $M_{A}=1.87$ ), subsonic (sonic Mach number: $M_{S}=0.82$ ) and submagnetosonic (magnetosonic Mach number: $M_{M S}=0.75$ ). Stationarity of the simulation is achieved after two passages of the magnetospheric plasma through the entire simulation domain, i.e. after about $644 \mathrm{~s}$. Please note that the end of the first simulation step (A) at $t=t_{1}$ takes place significantly later.

At $t=t_{1}$, the second step (B) of our real-time simulation is initiated. The boundary conditions at the left-hand side of the simulation domain are suddenly altered, representing the passage into a supermagnetosonic plasma regime. However, neither the magnitude nor the direction of the plasma bulk speed is changed. During the entire simulation, the upstream bulk speed is set to a value of $\underline{u}_{0}=(120,0,0) \mathrm{km} / \mathrm{s}$. This issue will be discussed in more detail in the next section. For $t_{1}<t<t_{2}=2 t_{1}$, the following input conditions have been applied to the $\left(x=-7.5 R_{T}\right)$ wall of the simulation domain: The magnetic field direction is reversed with respect to the situation inside the magnetosphere, i.e. we now use a value of $\underline{B}_{0}=(0,0,5) \mathrm{nT}$. The plasma entering the simulation box does no longer include an oxygen component, but the flow only consists of a cold hydrogen $\left(\mathrm{H}^{+}\right)$component of density $n\left(\mathrm{H}^{+}\right.$cold $)=3.2 \times 10^{6} \mathrm{~m}^{-3}$ and temperature $k T=12.5 \mathrm{eV}$. Thus, the values of the three Mach numbers in simulation step B are given by $M_{A}=1.97$ (superalfvénic), $M_{S}=2.19$ (supersonic) and $M_{M S}=1.46$ (supermagnetosonic). The thermal velocity of the hydrogen flow is three times smaller than the value of the magnetospheric plasma. The second part of the simulation ends at $t_{2}=2 t_{1}=2170 \mathrm{~s}$, i.e. after about three passages of the supermagnetosonic hydrogen flow through the simulation box.

For $t_{2} \leq t$ (step C), the plasma and magnetic field parameters of the flow entering the simulation domain are again switched to the values from the first part (A), thus mimicking Titan's return into a superalfvénic, yet subsonic and submagnetosonic plasma regime. After about two passages of the plasma through the box, a stationary state is achieved again. The topology of Titan's plasma environment in this state should be identical to the configuration obtained directly before the initiation of step $\mathrm{B}$.

To sum up the basic idea, in the first step (A) of our simulation, Titan is exposed to a submagnetosonic and hot magnetospheric plasma flow. When the second part of the simulation commences (B), the upstream parameters are changed in order to permit a real-time study of the transition that Titan's plasma environment undergoes when the satellite enters a regime of cold, supermagnetosonic hydrogen plasma, accompanied by a reversal of the ambient magnetic field direction. In the final part of the simulation (C), Titan re-enters the submagnetosonic plasma regime. This goes along with a return of the magnetic field orientation to its original direction. Again, the simulation shall allow to study in real-time the reconfiguration of Titan's induced magnetosphere. 


\subsection{Discussion of simulation technique}

In this article, we present the first application of a global simulation model to study in real-time the transition that Titan's plasma environment undergoes when the moon leaves the magnetosphere of its parent planet and enters a superfast solar wind flow. However, the reader should be aware of the simplifications that need to be included when conducting such a type of real-time hybrid simulation.

First and foremost, the velocity and direction of the impinging plasma flow remain the same throughout the entire duration of the simulation. The (numerical) reasons for this constraint are discussed in detail in our two companion papers on simulations of Titan's plasma interaction under nonstationary upstream conditions (Simon et al., 2008, 2009a). In reality, when Titan leaves Saturn's magnetosphere near noon local time due to enhanced solar wind dynamic pressure, the planet's magnetopause and bow shock move nearly perpendicular to the corotational flow direction inside the magnetosphere. Neither the velocities of Saturn's bow shock and magnetopause nor the velocity of the solar wind outside the magnetosphere need to be identical to the corotational flow speed of the magnetospheric plasma impinging on Ti$\tan$. This implies that the orientation of Titan's dayside ionosphere with respect to the direction of the impinging plasma may also change during an excursion into the solar wind.

Currently, the only possibility of self-consistently covering these effects would be the application of a multi-fluid model. By using such an approach, the response of Saturn's magnetosphere to a change in the solar wind conditions as well as the transition that the plasma signatures in Titan's immediate vicinity undergo could be studied simultaneously within the framework of a two-body simulation. Such a model could also consider that when Saturn's magnetosphere expands and Titan re-enters the region dominated by the planetary magnetic field, the magnetopause boundary layer approaches the moon from the wakeside. A multi-fluid model has already been used to study Titan's plasma environment during the TA, TB and T3 encounters (Snowden et al., 2007). However, Titan's plasma interaction during a solar wind excursion has not yet been analyzed by applying the multi-fluid approach. Besides, the reader should keep in mind that any kind of fluid-model fails in reproducing the effects associated with the large gyroradii of the involved pickup species. As demonstrated e.g. by Luhmann (1996) and Brecht et al. (2000), the influence of finite gyroradii makes up a key feature of the Titan interaction scenario. The importance of ion kinetic effects for understanding Titan's plasma interaction in the case of non-stationary upstream conditions (e.g. during a magnetopause passage) has been emphasized by Simon et al. (2008). In that work, the applicability of our model approach described above to the real Titan interaction scenario is also discussed in more detail.

In our model, the change in the upstream parameters is mimicked by a modification of the boundary conditions at the left-hand side of the simulation domain. Titan transfers directly from the submagnetosonic to the supermagnetosonic plasma regime. The moon's motion through Saturn's magnetopause (that possesses a finite thickness), magnetosheath and bow shock is not resolved. In other words, the assumption of a simple passage through a current sheet does not only ignore the spatial inhomogeneities of the transition region between magnetosphere and solar wind, but the duration that Titan needs in order to transfer from the subfast to the superfast flow regime is underestimated as well. In reality, Titan at first exits Saturn's magnetosphere and enters the magnetosheath, going along with a change in the plasma composition (from oxygen to hydrogen) and probably in the magnetic field direction. Subsequently, the moon makes a second transfer from subfast to superfast hydrogen flow regime by crossing the region of dense plasma and magnetic field lines in Saturn's bow shock. In our simulation scenario, these steps are "compressed" to a single transit through a current sheet.

However, the primary purpose of our simulation is to isolate those effects that emerge from the transition from the magnetospheric to a superfast flow regime. Therefore, we intend to keep the representation of the change in the upstream flow parameters as simple as possible. The parameters of the submagnetosonic flow regime in our simulation correspond to the well-understood Voyager 1 scenario, whereas - except for the temperature of the supersonic hydrogen flow - the upstream parameters in part B are nearly the same as those of the magnetosheath plasma in our recently published T32 simulation paper. Hence, the results of the preceding T32 study can provide an important reference for understanding the transition that Titan's induced magnetosphere undergoes when the moon enters a supermagnetosonic flow regime. Our intention of keeping the geometry as simple as possible is also the reason why we consider a complete magnetic field reversal, whereas the magnitude of $\underline{B}_{0}$ is left unchanged. Of course, if in-situ observations of this transition scenario become available, a refinement of the current simulation approach will most likely be required.

\section{Simulation results I: Titan leaves Saturn's magnetosphere}

The results of our real-time simulation are displayed in Figs. 3 to 16 . Similar to a flip-book, this sequence of snapshots illustrates the transition that Titan's plasma environment undergoes when the moon enters a supermagnetosonic flow regime and subsequently returns into the magnetospheric plasma of its parent body. For the $(y=0)$ plane of the coordinate system introduced in Fig. 1, each figure shows the number density of oxygen ions in the ambient flow in plot (a) and the number density of cold hydrogen ions in plot (b). The cold hydrogen ions are injected into the box only during step B of the real-time simulation. The evolution of the magnetic field topology in the vicinity of Titan can 

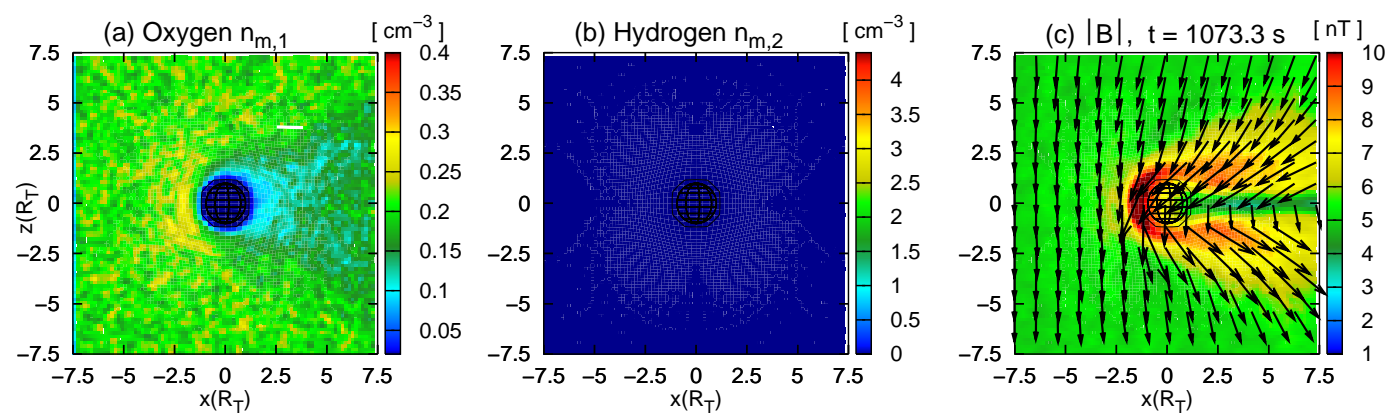

Fig. 3. Titan's plasma interaction during a solar wind excursion, flip-book of real-time simulation results (part A). The figure displays (a) the magnetospheric oxygen density, (b) the density of cold hydrogen ions and (c) the magnetic field strength and direction for the quasi-stationary state of the first simulation interval $\left(t \leq t_{1}\right)$.
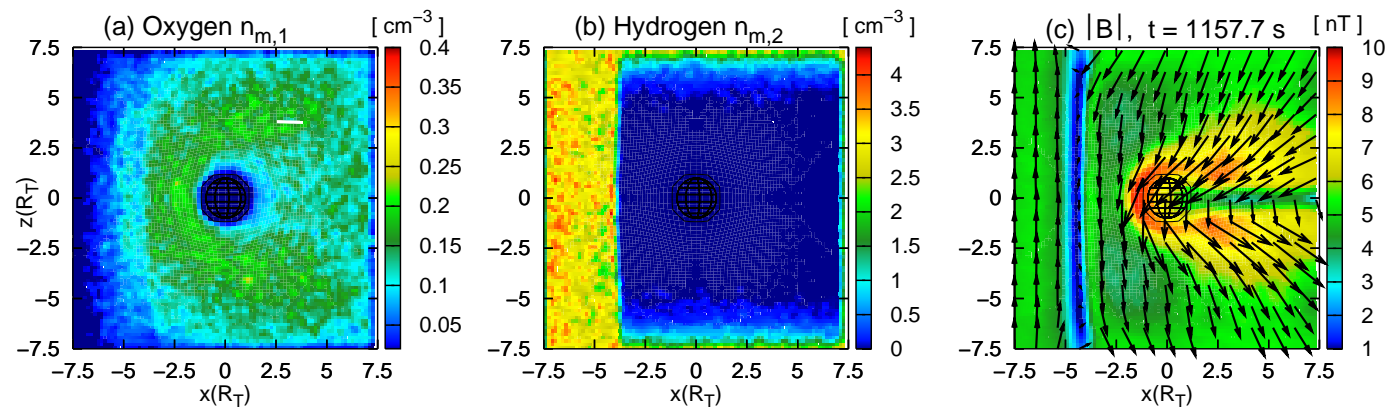

Fig. 4. Titan's plasma interaction during a solar wind excursion, flip-book of real-time simulation results (part B). The figure displays (a) the magnetospheric oxygen number density, (b) the number density of cold hydrogen ions and (c) the magnetic field directly after the entry of the supermagnetosonic hydrogen flow into the simulation box.
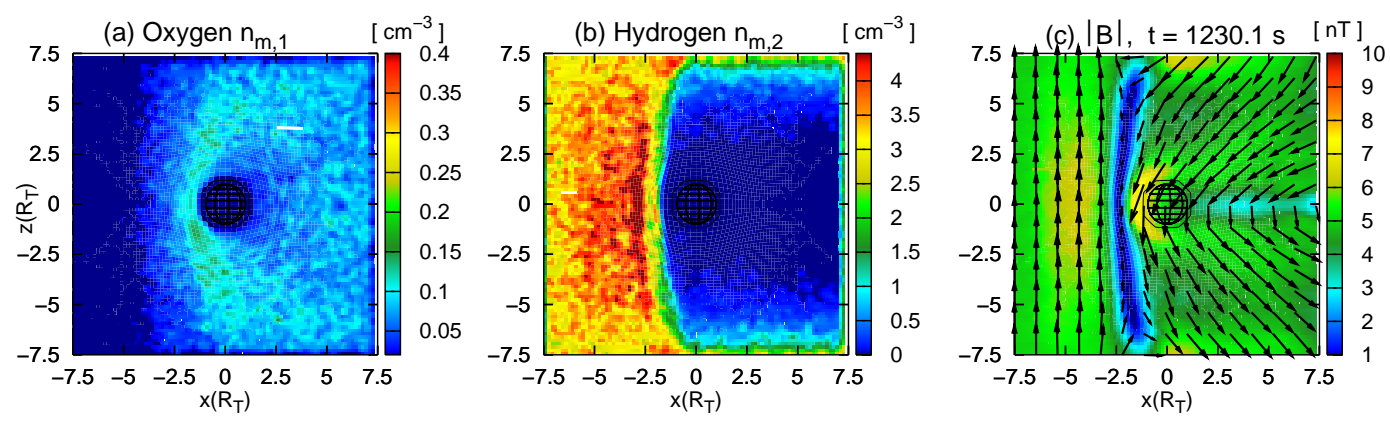

Fig. 5. Titan's plasma interaction during a solar wind excursion, flip-book of real-time simulation results (part B). The quantities displayed in the figure are the same as in Fig. 4. The diffuse increase of the hydrogen density upstream of Titan is a first hint towards the development of a shock front.

be seen in the sequence of (c) plots. The arrows denote the projection of the magnetic field vectors on the $(x, z)$ plane, while the color bar indicates the local field strength. As already demonstrated by Simon et al. (2007b), the dilute magnetospheric hydrogen component does not take noteworthy influence on the overall structure of the interaction region. Due to their small mass density, these particles merely act as test particles that are inserted into a given electromagnetic field configuration. Therefore, the density of the magnetospheric hydrogen population is not displayed here.
The structures in Titan's plasma environment right before $t=t_{1}$ are illustrated in Fig. 3. As can be seen from plot (c), the magnetic field lines form a pronounced draping pattern, featuring a magnetic pile-up region at the moon's dayside and two magnetic lobes in the wake region. Above Titan's dayside ionosphere, the background magnetic field strength is exceeded by more than a factor of 2 , whereas in the lobes, a peak field value of about $9 \mathrm{nT}$ is achieved. Our companion papers cited above contain more detailed discussions and illustrations of the magnetic field topology near Titan. Overall, 
the situation is also very similar to the magnetic environment of Mars or comets. Full 3-D illustrations of the field line topology for these scenarios, obtained from the same model, are provided by Bößwetter et al. (2004) and Bagdonat (2005). The oxygen density downstream of Titan is slightly reduced because these particles are partially deflected around the obstacle along the highly curved magnetic field lines. The configuration shown in Fig. 3 also represents the quasistationary state of the simulation at the end of step C.

The results for part B of the simulation are illustrated in plots 4 to 11. As can be seen from Fig. 11, in the quasistationary state of step B, Titan is embedded into the superfast hydrogen flow, with a parabolically shaped bow wave being clearly visible in the number density as well as in the magnetic field. The transition from subfast to superfast flow regime shall be discussed in the following. Figures 4 and 5 show how the discontinuity between both plasmas enters the simulation domain from the left-hand side and finally reaches the magnetic pile-up region above Titan's ramside ionosphere. As can be seen from plot $4 \mathrm{c}$, the magnetic fields upstream and downstream of the discontinuity point in opposite directions. The figure also illustrates the importance of the high temperature in the magnetospheric oxygen flow. Since the oxygen thermal velocity clearly exceeds the bulk speed of the plasma, a huge number of oxygen ions posses an individual velocity that is directed antiparallel to the bulk flow direction. These particles are able to cross the discontinuity between both plasma regimes from downstream to upstream, leading to a rather diffuse outer boundary of the oxygen population in the upstream region (cf. Fig. 4a).

When the discontinuity encounters the compressed magnetic field lines above Titan's ramside ionosphere, the original draping pattern is eroded due to reconnection (cf. Simon et al., 2009a, for details). However, the most important aspect becomes evident when comparing the wakeside magnetic field magnitudes in plots $4 \mathrm{c}$ and $5 \mathrm{c}$. The arrival of the oppositely directed magnetic field at Titan does not only erode the piled-up field lines above the satellite's ramside ionosphere, but the magnetic field topology in the entire wake region is affected as well. The highly draped magnetic field lines at the wakeside of Titan tend to relax, already exhibiting a more parabolic shape than in the original submagnetosonic situation. As can be seen in plot 5c, shortly after the initiation of the ramside reconnection process, the magnetic field strength in the wake region has returned to the background value of about $5 \mathrm{nT}$. Moreover, plots $5 \mathrm{~b}$ and c illustrate that the formation of a shock wave upstream of Ti$\tan$ has already been initiated at this stage: a diffuse region of increased hydrogen density manifests upstream of the moon. The magnetic field in the upstream region commences to enhance as well, although a curvature of the field lines is not yet visible (cf. left part of Fig. 5c).

As can be seen from the sequence of (c) plots in Figs. 6-8, the discontinuity between both plasma regimes becomes deformed due to the increased magnetic convection time in the dense, slow ionospheric plasma that surrounds Titan. Since the convection at the flanks of the interaction region (i.e. for $\left.|z| \gg R_{T}\right)$ is not affected, the discontinuity starts to exhibit a kink-like shape. This effect and its impact on the wakeside magnetic reconfiguration process are discussed in more detail in our companion study (Simon et al., 2009a). The formation of a bow wave ahead of Titan leaves a pronounced imprint on both the plasma density and the magnetic field topology. The initially broad and diffuse density enhancement steadily converges into a parabolically shaped shock front (cf. plots $6 \mathrm{~b}, 7 \mathrm{~b}$ and $8 \mathrm{~b}$ ). Simultaneously, the magnetic field lines in the upstream region start to curve and finally exhibit a parabolic shape as well. It is important to notice that the formation of the shock wave upstream of Titan is completed only a few minutes after the arrival of the supermagnetosonic plasma flow at the moon's ramside.

As shown in Figs. $7 \mathrm{~b}$ and $8 \mathrm{~b}$, the magnetic neutral sheet also separates the region filled by cold hydrogen ions from the area downstream of Titan: hydrogen ions can be found mainly upstream of the deformed discontinuity. In this stage, a pronounced density cavity is visible in the downstream region. Figure $7 \mathrm{~b}$ illustrates that directly behind Titan, the hydrogen density void exhibits a cylindrical shape, while further downstream, it broadens and transforms into a cone-like structure. The region filled by cold hydrogen ions seems to "drape" around Titan during the reconfiguration process. Subsequently, the wakeside density void is refilled from both sides and starts to collapse (cf. plots $9 \mathrm{~b}$ and 10b). As shown in Fig. 9b, the inner boundaries of the cylindrical wake get into contact at about $x=2.5 R_{T}$. The region of nearly vanishing hydrogen density is subsequently "detached" from Titan and travels downstream (cf. plot 10b). This process features a certain resemblance to the detachment of a plasmoid in the Terrestrial magnetotail.

In strong analogy to the transition from magnetosphere to magnetosheath investigated by Bertucci et al. (2008), the overall picture of the magnetic field topology is very similar to the situation near a comet that crosses a sector boundary in the solar wind. The boundary layer between the oppositely directed magnetic fields is opened due to reconnection, at first only at the ramside and subsequently in the wake region. The newly merged magnetic field lines that are no longer connected to Titan are well visible in Figs. 7c and $8 \mathrm{c}$. While the repolarization of the wakeside magnetotail still proceeds, the shock front at Titan's ramside is already fully established. The ramside magnetic field topology as well as the density pattern upstream of Titan do not exhibit any more significant changes until the end of simulation step B.

Plots $8 \mathrm{c}$ to $11 \mathrm{c}$ also illustrate that the magnetic field strength in the downstream region remains at its background value until the slightly deformed discontinuity has left the simulation domain. The "growth" of two new magnetic lobes (denoted by an increased field strength) at Titan's wakeside from above and below the satellite's poles commences after the wakeside magnetic repolarization process has been 

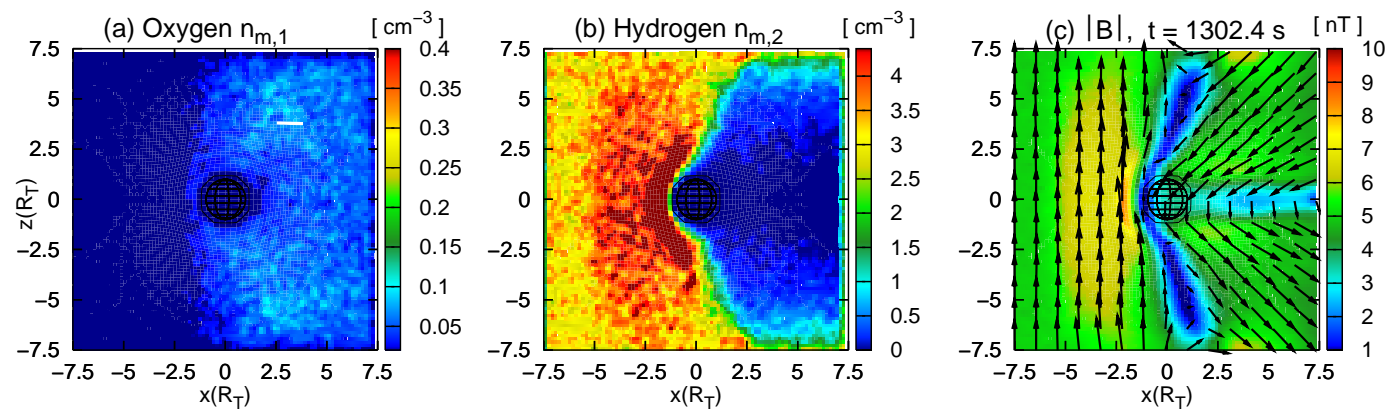

Fig. 6. Titan's plasma interaction during a solar wind excursion, flip-book of real-time simulation results (part B). The quantities displayed in the figure are the same as in Fig. 4. The parabolic structure of the bow shock already manifests in the plasma density as well as in the magnetic field strength.
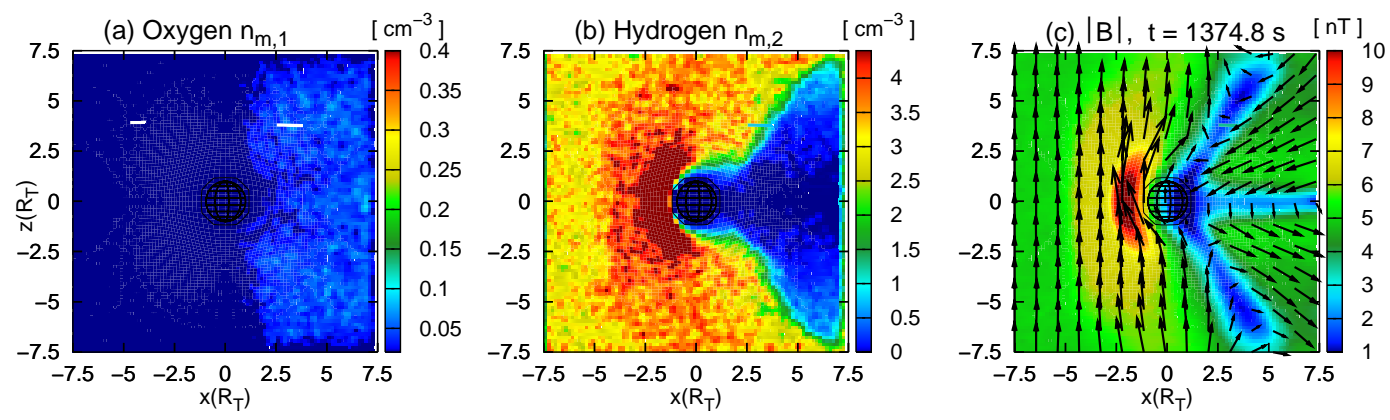

Fig. 7. Titan's plasma interaction during a solar wind excursion, flip-book of real-time simulation results (part B). The quantities displayed in the figure are the same as in Fig. 4. The density void downstream of Titan is not refilled instantaneously, but a cylindrical wake cavity is formed.
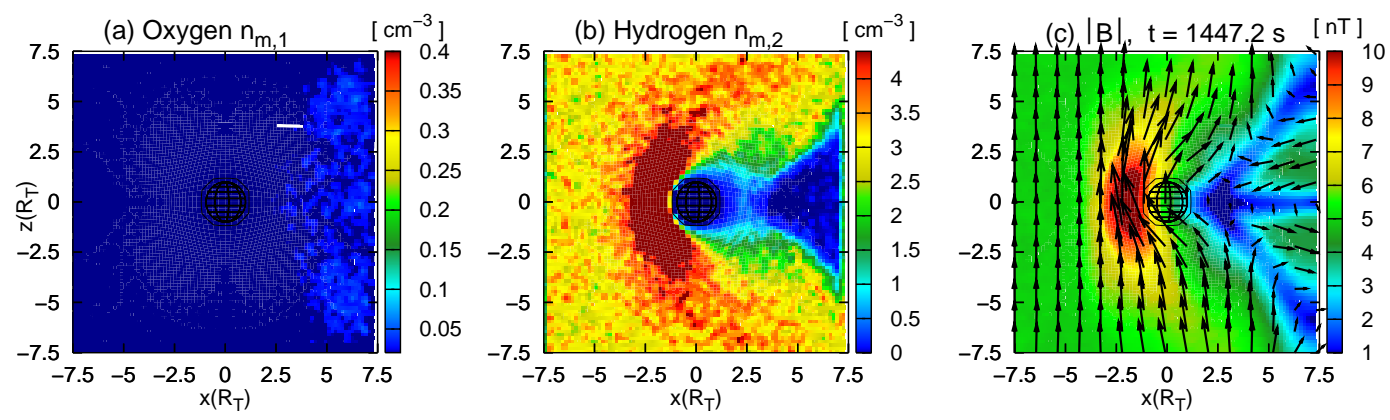

Fig. 8. Titan's plasma interaction during a solar wind excursion, flip-book of real-time simulation results (part B). The quantities displayed in the figure are the same as in Fig. 4. The build-up of the shock front at Titan's ramside is complete. The repolarization of the wakeside magnetotail due to reconnection is still in progress.

completed. The final state of the magnetic field topology is shown in Fig. 11c. Since the curvature of the parabolically shaped field lines is weaker than in the submagnetosonic situation, a peak field magnitude of only about $7 \mathrm{nT}$ is achieved in the wakeside magnetic lobes. To sum up, the reconfiguration of the magnetic field topology takes place in three subsequent steps: Shortly after ramside reconnection has been initiated, the magnetic field lines at Titan's wakeside are relaxed, with the field strength in the downstream region drop- ping to the background value of about $5 \mathrm{nT}$. Subsequently, the polarity of Titan's magnetotail is reversed. During this process, the magnetic field strength in the entire wake region does not exceed the undisturbed background field value. After the polarity of the wakeside magnetotail has been reversed, the field lines are compressed again and two new magnetic lobes are formed. In strong analogy to the T32 scenario (Bertucci et al., 2008; Simon et al., 2009a), the simulation results suggest only a few minutes of retardation 

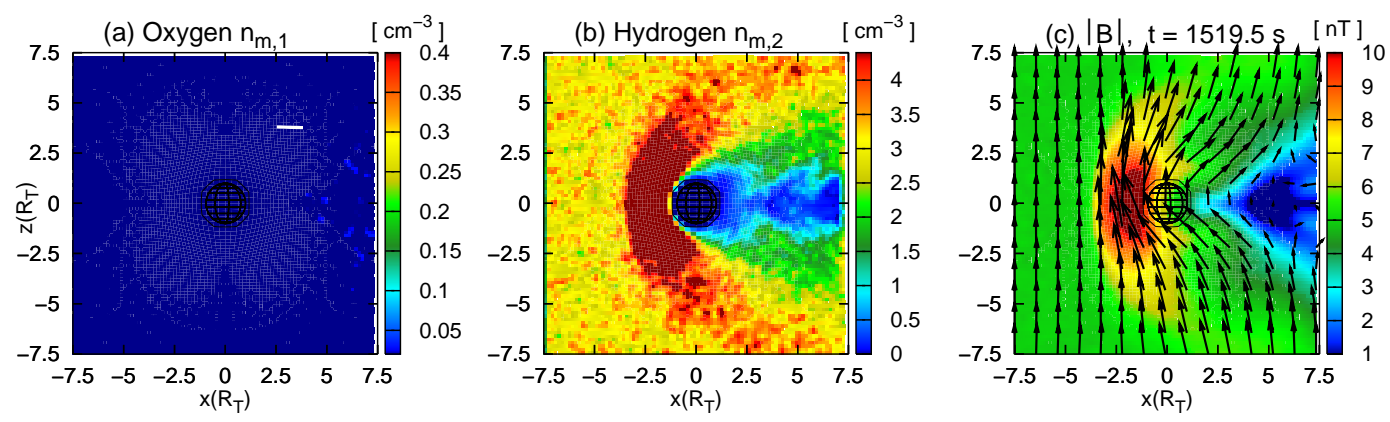

Fig. 9. Titan's plasma interaction during a solar wind excursion, flip-book of real-time simulation results (part B). The quantities displayed in the figure are the same as in Fig. 4. A cone-like region of reduced hydrogen density arises downstream of Titan.
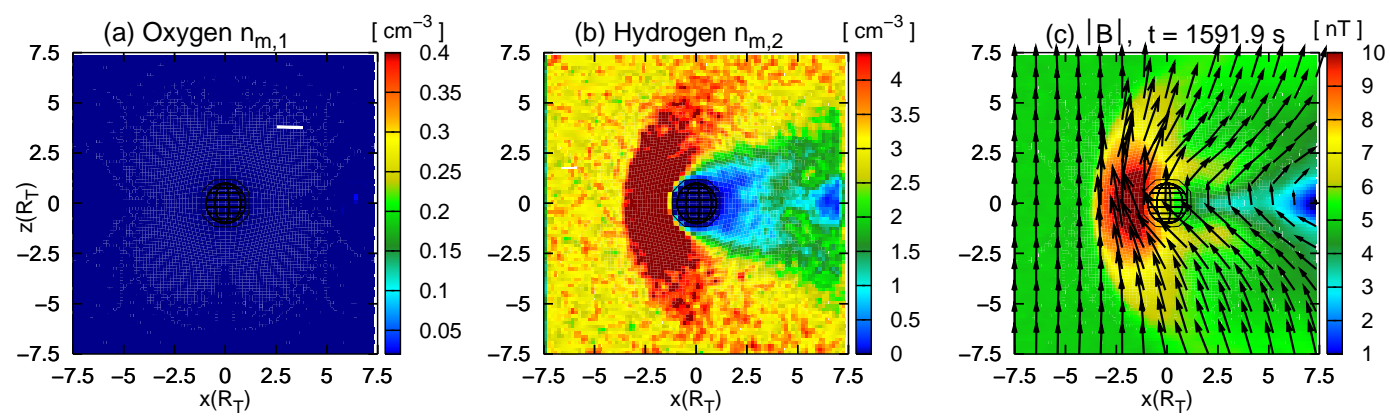

Fig. 10. Titan's plasma interaction during a solar wind excursion, flip-book of real-time simulation results (part B). The quantities displayed in the figure are the same as in Fig. 4. After the magnetic reconfiguration process in Titan's wake has been completed, two new magnetic lobes (characterized by an increased field strength) start to "grow" near the north and south poles of the moon.
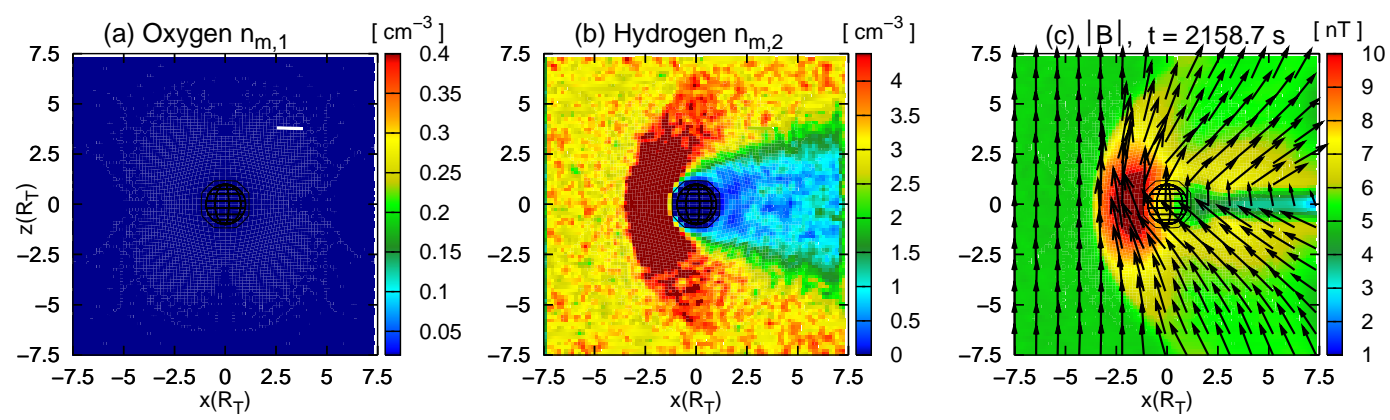

Fig. 11. Titan's plasma interaction during a solar wind excursion, flip-book of real-time simulation results (part B). The figure displays (a) the magnetospheric oxygen density, (b) the density of cold hydrogen ions and (c) the magnetic field strength and direction for the quasistationary state of the second simulation interval (i.e. short before $t=t_{2}$ ). A peak field strength of about $7 \mathrm{nT}$ is achieved in the wakeside magnetic lobes.

between the traveling of the discontinuity through the simulation domain and the finalization of the repolarized magnetotail topology.

\section{Simulation results II: Titan re-enters Saturn's magnetosphere}

In this section, the results from part $\mathrm{C}$ of our real-time simulation shall be discussed. At $t=t_{2}$, the boundary conditions at the left-hand side of the simulation box are reconfigured again: The magnetic field direction is reversed, while simultaneously, the submagnetosonic magnetospheric plasma flow re-enters the simulation domain and replaces the cold hydrogen population (cf. Fig. 12). The results of this final step are shown in the second part of the flip-book (plots 12 to 16).

The shock front ahead of Titan is characterized by a broad, parabolically shaped magnetic field enhancement. Even at $x=-3 R_{T}$, the magnetic field strength exceeds the 

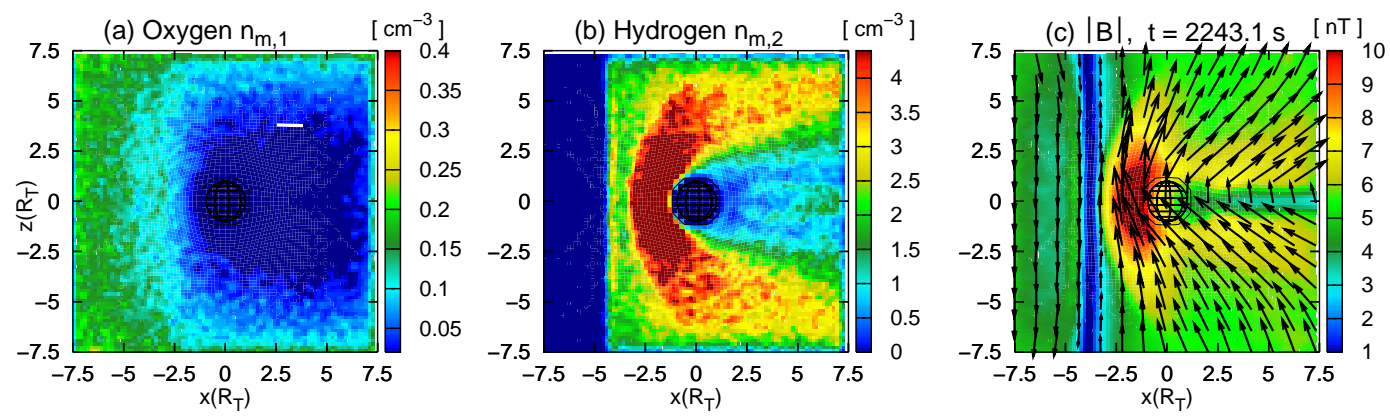

Fig. 12. Titan's plasma interaction during a solar wind excursion, flip-book of real-time simulation results (part C). The quantities displayed in the figure are the same as in Fig. 4. The plots illustrate the structure of Titan's plasma environment after the boundary parameters at the left-hand side of the box have been switched to magnetospheric conditions again.
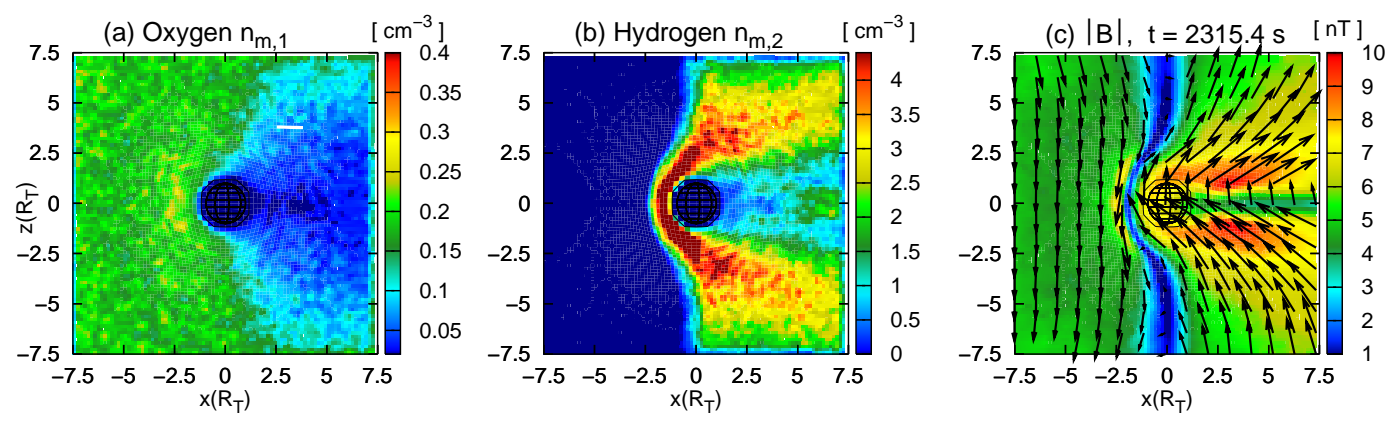

Fig. 13. Titan's plasma interaction during a solar wind excursion, flip-book of real-time simulation results (part C). The quantities displayed in the figure are the same as in Fig. 4. The broad magnetic field enhancement in the shock front has already been eroded. The dense hydrogen plasma from the shock front is pressed into the wake region.
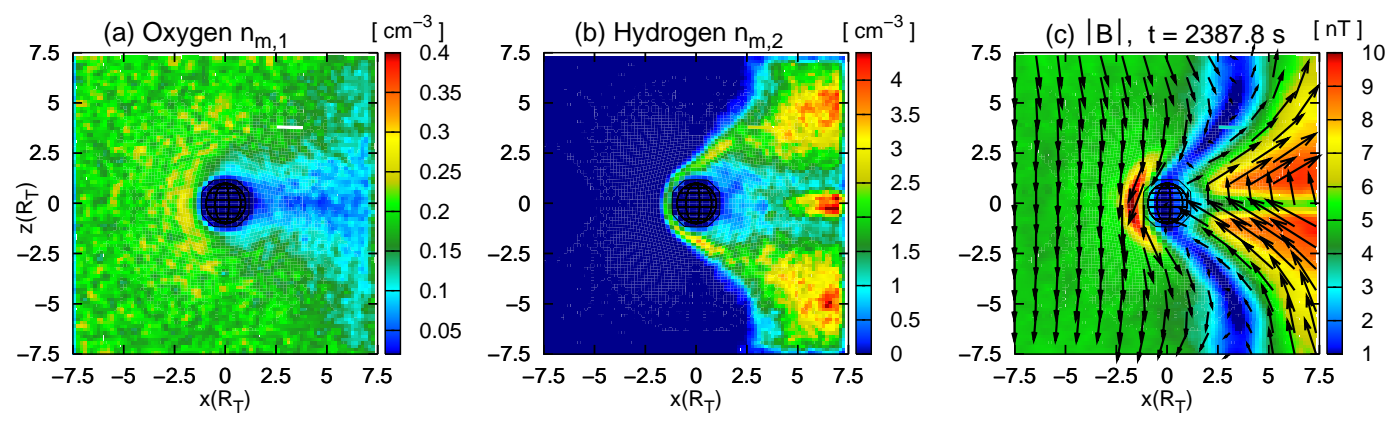

Fig. 14. Titan's plasma interaction during a solar wind excursion, flip-book of real-time simulation results (part C). The quantities displayed in the figure are the same as in Fig. 4. The hydrogen density plot shows that the remnants of the shock front have been completely disconnected from the satellite.

background value by almost a factor of 2 . This broad region of increased field magnitude is removed, once the magnetospheric plasma flow has arrived at Titan's ramside. Since practically no newly generated pick-up ions can be found at large distances upstream of Titan, the convection time of the magnetic field remains unaffected until the discontinuity has arrived above the ramside ionosphere of the moon. Therefore, the discontinuity between supermagnetosonic and submagnetosonic plasma regime does not experience a deforma- tion during the erosion of the piled-up magnetic field lines in the shock front. The curvature of the discontinuity sets in after it has entered the slow ionospheric plasma in the immediate vicinity of Titan, cf. Fig. 13c. During the ramside magnetic reconfiguration process, the draped field lines in Titan's wake are initially compressed. As can be seen from Figs. 13c and 14c, the magnetic field strength in Titan's wake does not only exceed the maximum value that is achieved in the supermagnetosonic case, but it is even larger than the wakeside 

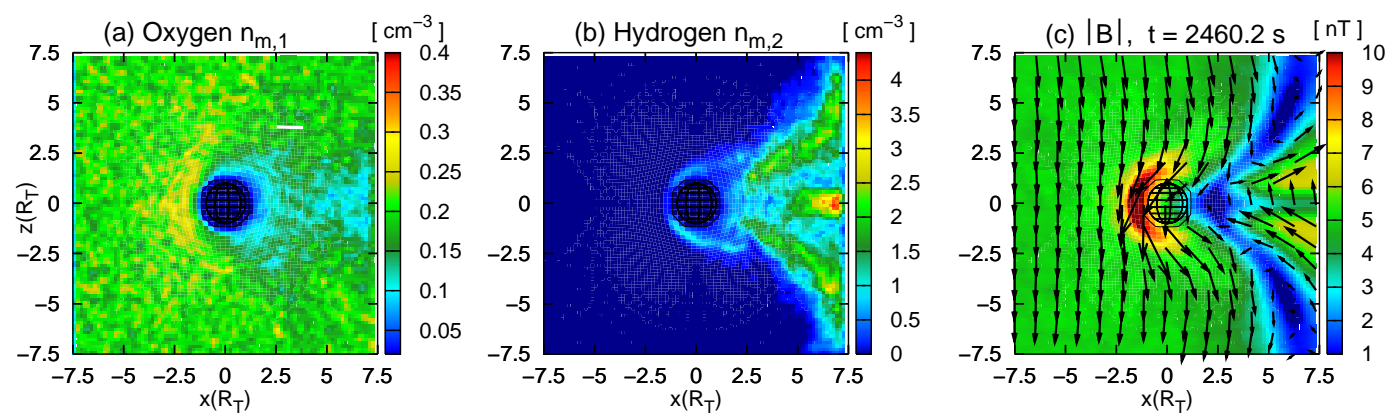

Fig. 15. Titan's plasma interaction during a solar wind excursion, flip-book of real-time simulation results (part C). The quantities displayed in the figure are the same as in Fig. 4. The magnetic pile-up region at Titan's ramside has already achieved its final shape, while the reconfiguration of the wakeside magnetotail is still proceeding. Newly merged magnetic field lines that are not connected to Titan are well visible along the flanks of the discontinuity.
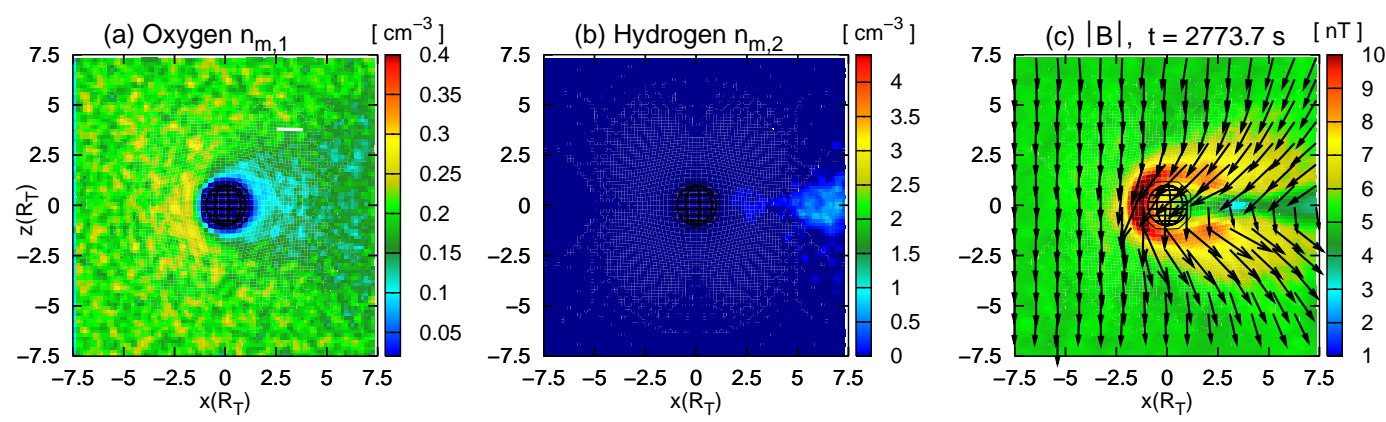

Fig. 16. Titan's plasma interaction during a solar wind excursion, flip-book of real-time simulation results (part C). The figure displays (a) the magnetospheric oxygen density, (b) the density of cold hydrogen ions and (c) the magnetic field strength right after the discontinuity has left the simulation domain. The magnetic lobes are not yet fully developed, but the situation is already close to the final state of the simulation displayed in Fig. 3.

peak field magnitude in the quasi-stationary state of step A (cf. Fig. 3c). A very similar effect occurs during Titan's passage from the magnetospheric plasma to another submagnetosonic flow regime in Saturn's magnetosheath (Simon et al., 2009a). The repolarization of the wakeside magnetotail due to reconnection is highly analogous to the magnetospheremagnetosheath transition observed during the T32 encounter, i.e. the qualitative features of the reconfiguration process are not affected by the superfast nature of the impinging hydrogen flow.

In the final state of the transition from subfast to superfast flow regime (i.e. at the end of simulation step B), the field magnitude in the wakeside lobes is smaller than the value achieved in the initial tail structure inside the magnetosphere. However, before this state is achieved, the magnetic field lines tend to "overshoot": the initial relaxation of the wakeside field lines leads to a reduction of the field magnitude below the final value that is reached in the lobes at the end of step B. This final value is achieved after a subsequent compression of the field lines. A very similar "overshoot effect" in the wakeside magnetotail structure seems to occur during the transition from superfast to subfast flow regime.
When being located inside Saturn's magnetosphere, the field strength in Titan's magnetotail exceeds the peak field value that is achieved in the supermagnetosonic upstream situation. However, when the discontinuity arrives at Titan's ramside, the initial compression of the field lines leads to an even larger wakeside field magnitude than in the final state of step C. The final configuration of the lobes in the quasistationary state of step $\mathrm{C}$ is achieved by a subsequent drop of the field strength.

Simultaneously to the magnetic field reconfiguration, the density enhancement in the shock front is eroded during the passage of the discontinuity through the upstream region. The dense hydrogen plasma from the shock front is deflected around Titan, leading to an increased number density in the wake of the satellite (cf. Fig. 13b). As shown in Fig. 14b, the region of increased hydrogen density is finally split up near the subsolar point above Titan's ramside ionosphere. The remnants of the shock completely detach from Titan and travel downstream. It is interesting to notice that the boundary between northward and southward magnetic field also confines the cold hydrogen ions to the downstream region. The stronger the deformation of the discontinuity due to the 
enlarged convection time in Titan's ramside ionosphere, the smaller is the opening angle of the cone-like region populated by hydrogen ions, cf. plots $13 \mathrm{~b}$ to $15 \mathrm{~b}$. Again, the simulation results suggest the entire reconfiguration process to take place on a characteristic time scale of less than $10 \mathrm{~min}$.

Although the results are not shown here, we have also investigated the influence of the change in the upstream conditions on the structure of Titan's exospheric plasma tail. As demonstrated e.g. by Simon et al. (2007b), the tails developed by the three ionospheric species included into the model are shifted in the direction of the convective electric field, with their extension perpendicular to the upstream flow direction depending linearly on the mass of the respective ion species. In our preceding studies of Titan's plasma environment under non-stationary upstream conditions, it has been shown that Titan's plasma tail does not experience a detachment from the satellite, when

- Titan encounters a reversal of the ambient magnetic field direction, whereas the field magnitude and the plasma composition are left unchanged.

- the ambient magnetic field changes its direction and magnitude. Simultaneously, the composition of the upstream flow is switched from magnetospheric oxygen to thermalized hydrogen ions. This was the case during the T32 magnetopause crossing.

In the simulation scenario discussed in this article, we additionally reduced the temperature of the impinging plasma in order to mimic a supermagnetosonic upstream situation. The reorientation of Titan's exospheric plasma tail has shown to be highly analogous to the scenario discussed in detail by Simon et al. (2008). The tail is shifted in the direction of the reversed convective electric field, but it does not get disconnected from the satellite. This statement is valid for Titan's transit into the solar wind as well as the subsequent return into the subfast flow regime. As discussed in our companion studies, the large gyroradii of the heavy $\mathrm{N}_{2}^{+}$and $\mathrm{CH}_{4}^{+}$ions play a key role for understanding the evolution of the satellite's plasma tail during the reconfiguration process.

In addition to the simulation discussed above, we have considered an ancillary test scenario. For this model case, only the change of the plasma composition (from hot oxygen to thermalized hydrogen) at the boundary layer has been included, whereas the magnetic field direction remained the same throughout the entire duration of the simulation. In this way, it is possible to isolate the effects that must be ascribed to the increased upstream Mach numbers from the additional influence of the magnetic field reversal. The results of this test run have shown that the build-up and subsequent removal of the shock front at Titan's ramside occur on practically the same time scales as in the scenario presented above. Key features of the cold hydrogen flow pattern - such as the collapse of the wakeside plasma void during the solar wind entry and the deflection of the dense plasma from the shock around Ti- tan during the subsequent return into the magnetosphere can be found in this scenario as well.

Finally, we shall discuss several numerical challenges associated with hybrid simulations of Titan's plasma interaction. Due to the necessity to kinetically represent the ion distribution function and therefore, to store the phase space data of millions of individual particles, only a limited number of about 90 grid cells is available in each spatial direction. One therefore has to find an adequate compromise between a simulation domain that is sufficiently large to contain most of the plasma signatures formed in the vicinity of the obstacle and a box that retains an adequate grid resolution to resolve ion dynamics. In the simulation presented here, an average grid resolution of $430 \mathrm{~km}$ is achieved. Although the curvilinear grid is adapted to the spherical geometry of the obstacle, its spatial resolution does not change significantly between different regions of the simulation domain (cf. illustration of the grid structure in Fig. 4 of Simon et al., 2006b). The characteristic length scales of oxygen motion are comparable to the gyroradius $r_{g}=u_{0} m / e B_{0}=4000 \mathrm{~km}$. These scales are well resolved by the simulation grid. However, it is important to notice that the resolution of $\mathrm{H}^{+}$ion dynamics is by far not that good: The corresponding typical length scales of $r_{g}=4000 / 16 \mathrm{~km}=250 \mathrm{~km}$ are of the same order as the resolution limit of the grid. If an even significantly coarser grid was applied, an adequate resolution of $\mathrm{H}^{+}$ion dynamics could no longer be guaranteed. The time step applied in the simulation is smaller than the gyration periods of all ion species included in the scenario. Therefore, for all involved species, the characteristic time scales of ion motion are fully resolved by the model.

When developing the simulation scenario, a box with a smaller size of $\pm 4 R_{T}$ (i.e. higher grid resolution) has been tested as well. The macroscopic features of the shock front have shown to be the same as in the results presented here. However, this box is too small to keep the propagation of the discontinuity at the outer flanks of the interaction region unaffected by the boundary conditions applied to the upper and lower faces of the simulation box.

On the other hand, even the large $\left( \pm 7.5 R_{T}\right)$ simulation domain is still too small to allow a study of the quasi-parallel shock regime which may be formed at the outer flanks of the interaction region, as well as associated features like upstream ion beams and ULF waves. In any case, it is doubtful whether these features could be fully resolved by the relatively coarse grid of a 3-D simulation approach. One- and two-dimensional hybrid codes, which allow a significantly higher grid resolution as well as a larger number of particles per cell than any 3-D model, have been successfully applied to study these features (e.g. Omidi et al., 1990). The size of the simulation domain chosen for the present study can be considered a compromise between achieving a reasonable grid resolution and simultaneously, containing a sufficiently large sector of the interaction region. 
(a) Interval B, magnetic field

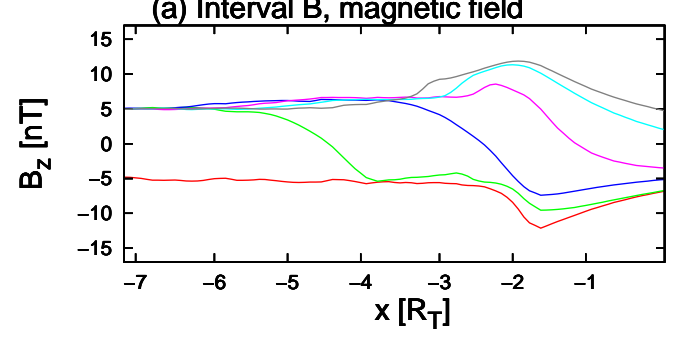

(b) Interval B, oxygen density

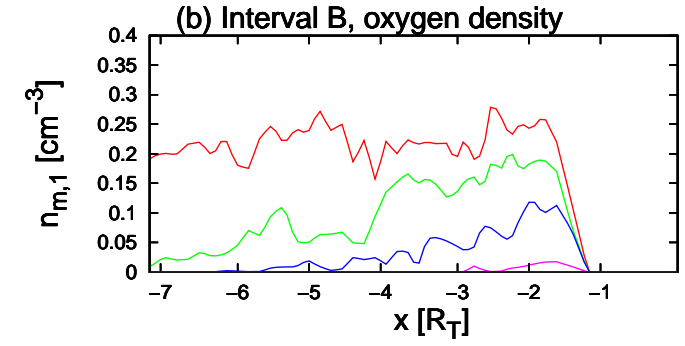

(c) Interval B, hydrogen density

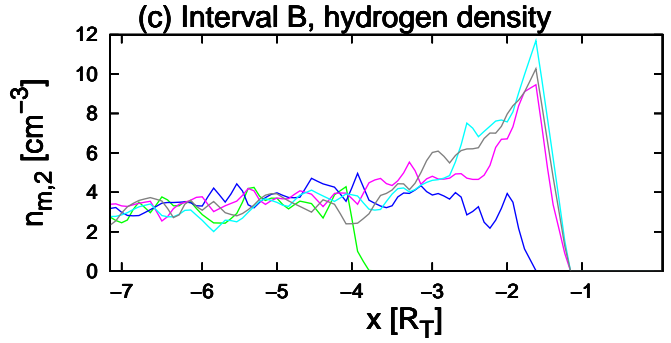

(d) Interval C, magnetic field

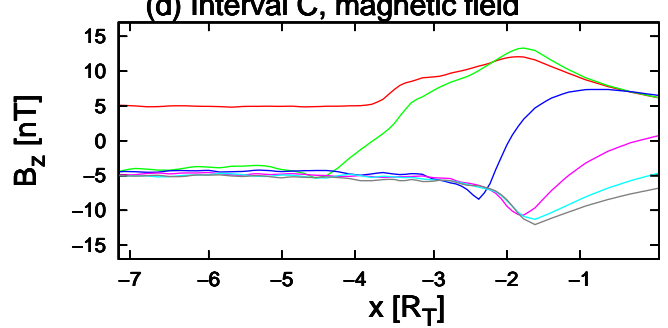

(e) Interval C, oxygen density
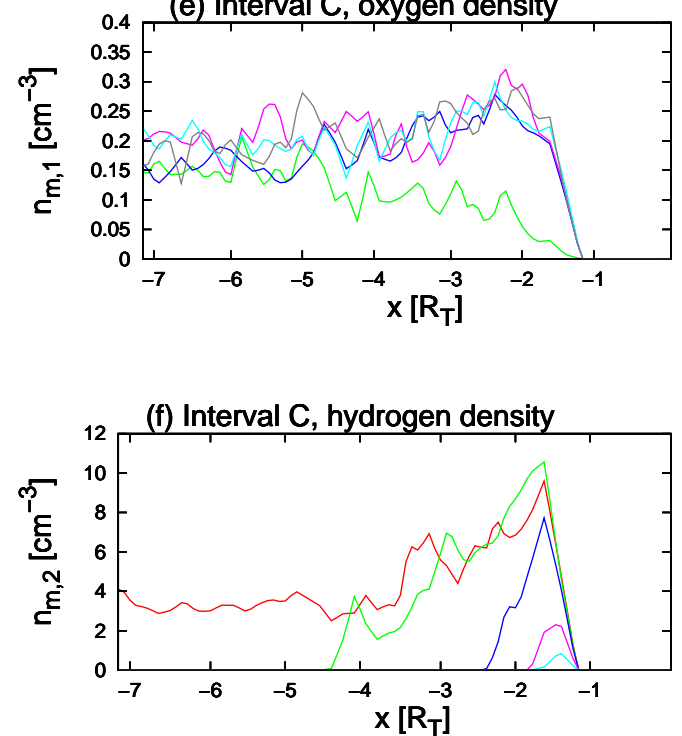

Fig. 17. 1-D density and magnetic field profiles. The figure displays a series of one-dimensional cuts along the $x$ axis $(y=z=0)$ upstream of Titan. The panels in the left-hand column refer to the situation in simulation interval B, i.e. to the transition from subfast to superfast upstream flow. Panel (a) displays the magnetic $B_{z}$ component, whereas plots (b) and (c) show the evolution of the oxygen and hydrogen density patterns, respectively. Different colors correspond to different points in time. Red: $t=1073.3 \mathrm{~s}$ (cf. Fig. 3), green: $t=1157.7 \mathrm{~s}$ (cf. Fig. 4), dark blue: $t=1230.1 \mathrm{~s} \mathrm{(cf.} \mathrm{Fig.} \mathrm{5),} \mathrm{magenta:} t=1302.4 \mathrm{~s} \mathrm{(cf.} \mathrm{Fig.} \mathrm{6),} \mathrm{light} \mathrm{blue:} t=1374.8 \mathrm{~s}$ (cf. Fig. 7), grey: $t=1447.2 \mathrm{~s}$ (cf. Fig. 8 ). The situation after the shock front has been fully established is illustrated by the red lines in the panels at the right-hand side. These plots show the same quantities for simulation interval C. The color coding in panels (d), (e) and (f) is as follows: Red: $t=2158.7 \mathrm{~s}$ (cf. Fig. 11), green: $t=2243.1 \mathrm{~s}$ (cf. Fig. 12), dark blue: $t=2315.4 \mathrm{~s} \mathrm{(cf.} \mathrm{Fig.} \mathrm{13),} \mathrm{magenta:} t=2387.8 \mathrm{~s}$ (cf. Fig. 14), light blue: $t=2460.2 \mathrm{~s}$ (cf. Fig. 15 ), grey: $t=2773.7 \mathrm{~s} \mathrm{(cf.} \mathrm{Fig.} \mathrm{16).} \mathrm{The} \mathrm{final} \mathrm{state} \mathrm{of} \mathrm{the} \mathrm{simulation} \mathrm{is} \mathrm{again} \mathrm{illustrated} \mathrm{by} \mathrm{the} \mathrm{red} \mathrm{lines} \mathrm{in} \mathrm{panels} \mathrm{(a),} \mathrm{(b)} \mathrm{and} \mathrm{(c).} \mathrm{Cuts} \mathrm{through}$ Figs. 9 and 10 are not shown, since at $t=1447.2 \mathrm{~s}$ (cf. Fig. 8), the structures at the ramside are already practically stationary.

\section{Summary and concluding remarks}

The plasma environment of Saturn's largest moon Titan has shown to be highly variable. Since Titan's orbit is located within the outer magnetosphere of Saturn, the moon can leave the magnetic field of the giant planet in times of high solar wind dynamic pressure and interact with the shocked plasma in Saturn's magnetosheath or even with the unshocked solar wind. The former situation has been observed for the first time during the Cassini T32 encounter of Titan which took place in June 2007. During this flyby, the Cassini magnetometer detected an almost complete reversal of the ambient magnetic field direction. The composition of the impinging plasma also changed dramatically. The tran- sition that Titan's plasma environment undergoes in such a non-stationary upstream situation has recently been analyzed by considering both Cassini magnetometer data and numerical modeling.

Although currently, no in-situ observations of such a scenario are available, Bertucci et al. (2008) suggest that a few hours before the T32 flyby, Titan might have been exposed to the supermagnetosonic, undisturbed solar wind upstream of Saturn. Following recent numerical modeling attempts of Titan's plasma interaction during the T32 magnetosheath excursion, this paper presents a first study of the transition that the moon's plasma environment undergoes during an excursion into the superfast solar wind and a subsequent return into the magnetospheric plasma regime. Our analysis 
is based upon a three-dimensional, electromagnetic hybrid code, treating the ions of the plasma as individual particles. The electrons form a massless, charge-neutralizing fluid. In order to isolate the effects that arise from the change in the upstream Mach numbers, the transition between submagnetosonic magnetospheric plasma and superfast solar wind flow has been realized by a simple modification of the boundary conditions at the left-hand side of the simulation domain: On the one hand, the direction of the ambient magnetic field is reversed. On the other hand, the hot magnetospheric oxygen plasma is replaced by a cold, superfast hydrogen population. Titan's return into the original submagnetosonic plasma regime has been studied as well. Currently, no spacecraft data are available for a quantitative comparison to our simulation results. Therefore, the input parameters of the model have been chosen as close as possible to our preceding studies of Titan's plasma interaction under non-stationary upstream conditions, the purpose being to allow an easy qualitative comparison of the results.

In the first step of the simulation, we have investigated the transition that Titan's plasma environment undergoes when the moon leaves Saturn's magnetosphere and makes an excursion into a supermagnetosonic plasma flow. Once the discontinuity between both plasma regimes has reached the ramside ionosphere of Titan, the field polarity in the magnetic pile-up region starts to reverse. Even in this early stage, the entire magnetic field topology at the moon's wakeside is affected as well. The highly draped magnetic field lines start to relax, and the wakeside field strength drops to the value in the undisturbed upstream plasma flow. Due to the increased magnetic convection time in Titan's ramside ionosphere, the boundary between subfast and superfast plasma is slowed down in the immediate vicinity of the satellite and therefore starts to exhibit a kink-like shape. However, the global topology of Titan's induced magnetosphere is reconfigured on a characteristic time scale of only a few minutes. In strong analogy to the recently investigated transition from magnetosphere to magnetosheath, the duration of the reconfiguration is mainly determined by the convection of the magnetic field in the undisturbed plasma flow at the flanks of the interaction region. Once the superfast hydrogen flow has arrived at the ramside of Titan, a broad and diffuse region of increased plasma density and magnetic field strength arises upstream of the satellite. These structures converge into a parabolically shaped, sharply confined shock front ahead of the moon, with the total duration of this process being of the order of about $5 \mathrm{~min}$. When the upstream flow is supermagnetosonic, a cone-like region of reduced plasma density forms downstream of Titan.

In the second part of our real-time simulation, we have investigated Titan's re-entry into the magnetospheric plasma regime. The broad, parabolically shaped shock structure in the magnetic field ahead of Titan is removed almost instantaneously, since there are no slow ionospheric particles upstream of the moon that could decelerate magnetic field con- vection. The hot, dense plasma from the shock front is deflected around Titan, leading to an increase of the hydrogen density at the wakeside. Finally, the remnants of the shock front detach from the satellite and travel downstream. While the magnetic field lines in the wakeside lobes are initially compressed, their repolarization due to magnetic reconnection is highly analogous to the transition that takes place during a magnetosheath excursion. In both parts of the simulation scenario, the magnetic reconfiguration features a strong qualitative resemblance to the passage of a comet through a sector boundary in the solar wind. Neither the entry into the solar wind nor its return into the submagnetosonic magnetospheric regime lead to a detachment of Titan's exospheric tail.

Figure 17 shows a series of one-dimensional cuts through the simulation domain along the $x$ axis, illustrating in summary the major findings of this study. Panel (a) shows how the initial magnetic pile-up region at Titan's ramside $\left(B_{z}<0\right)$ is eroded and subsequently replaced by a bundle of oppositely directed field lines. Simultaneously, the magnetospheric oxygen flow leaves the simulation domain (cf. panel b). After having entered the simulation box, the hydrogen density starts to pile-up at the ramside of the obstacle, and a broad shock front is formed (cf. panel c). In the final part of the simulation, the magnetic field returns to its original direction (cf. panel d). The oxygen flow re-enters the simulation domain, but there is no pronounced density increase upstream of Titan (cf. panel e). The shock front upstream of Titan is removed on a characteristic time scale of only a few minutes, and the hydrogen flow leaves the simulation box (cf. panel f).

From the modeling point of view, the major weakness of the hybrid approach is still the lack of a sufficient resolution in Titan's ionosphere, thus forbidding the inclusion of complex chemical processes. This may be the major reason why so far, none of the existing hybrid codes succeeded in reproducing fossilized magnetic fields that can get trapped in Titan's collisional ionosphere during a change of the upstream field direction (Neubauer et al., 2006; Bertucci et al., 2008). However, even though MHD and multi-fluid models provide a sufficiently high resolution and are therefore able to reproduce this type of magnetic fine structure, they do not consider the large gyroradii of the pick-up ions. Probably, results from both fluid and kinetic modeling must be considered in order to fully understand the influence of non-stationary upstream conditions on Titan's plasma interaction.

Acknowledgements. The author is grateful to Uwe Motschmann (Institute for Theoretical Physics, TU Braunschweig), Joachim Saur and especially Fritz M. Neubauer (Institute of Geophysics and Meteorology, University of Cologne) for helpful discussions. Financial support by the Deutsche Forschungsgemeinschaft (DFG) under grant MO539/15-1 is also acknowledged.

Topical Editor I. A. Daglis thanks N. Omidi and another anonymous referee for their help in evaluating this paper. 


\section{References}

Backes, H.: Titan's Interaction with the Saturnian Magnetospheric Plasma, PhD thesis, Universität zu Köln, 2005.

Backes, H., Neubauer, F. M., Dougherty, M. K., Achilleos, N., André, N., Arridge, C. S., Bertucci, C., Jones, G. H., Khurana, K. K., Russell, C. T., and Wennmacher, A.: Titan's Magnetic Field Signature During the First Cassini Encounter, Science, 308, 992-995, 2005.

Bagdonat, T.: Hybrid Simulation of Weak Comets, Ph.D. thesis, Technische Universität Braunschweig, 2005.

Bagdonat, T. and Motschmann, U.: From a weak to a strong comet - 3D global hybrid simulation studies, Earth, Moon and Planets, 90, 305-321, 2002.

Bertucci, C., Achilleos, N., Dougherty, M. K., Modolo, R., Coates, A. J., Szego, K., Masters, A., Ma, Y., Neubauer, F. M., Garnier, P., Wahlund, J.-E., and Young, D. T.: The Magnetic Memory of Titan's Ionized Atmosphere, Science, 321, 1475, 2008.

Bößwetter, A., Bagdonat, T., Motschmann, U., and Sauer, K.: Plasma boundaries at Mars: a 3-D simulation study, Ann. Geophys., 22, 4363-4379, 2004, http://www.ann-geophys.net/22/4363/2004/.

Bößwetter, A., Simon, S., Bagdonat, T., Motschmann, U., Fränz, M., Roussos, E., Krupp, N., Woch, J., Schüle, J., Barabash, S., and Lundin, R.: Comparison of plasma data from ASPERA3/Mars-Express with a 3-D hybrid simulation, Ann. Geophys., 25, 1851-1864, 2007,

http://www.ann-geophys.net/25/1851/2007/.

Brecht, S. H., Luhmann, J. G., and Larson, D. J.: Simulation of the Saturnian magnetospheric interaction with Titan, J. Geophys. Res., 105, 13119-13130, 2000.

Coates, A. J., McAndrews, H. J., Arridge, C. S., Jones, G. H., Crary, F. J., Young, D. T., Szego, K., Sittler, E. C., Thomsen, M. F., Tokar, R. L., Bertucci, C., and Dougherty, M. K.: Titan at Saturn's magnetopause: CAPS results from T32, AGU Fall Meeting Abstracts, pp. A1021+, 2007.

Dougherty, M. K., Khurana, K. K., Neubauer, F. M., Russell, C. T., Saur, J., Leisner, J. S., and Burton, M. E.: Identification of a Dynamic Atmosphere at Enceladus with the Cassini Magnetometer, Science, 311, 1406-1409, doi:10.1126/science.1120985, 2006.

Kallio, E., Sillanpää, I., and Janhunen, P.: Titan in subsonic and supersonic flow, Geophys. Res. Lett., 31, L15703/1-L15703/4, doi:10.1029/2004GL020344, 2004.

Kallio, E., Sillanpää, I., Jarvinen, R., Janhunen, P., Dougherty, M., Bertucci, C., and Neubauer, F.: Morphology of the magnetic field near Titan: Hybrid model study of the Cassini T9 flyby, Geophys. Res. Lett., 34, 24, doi:10.1029/2007GL030 827, 2007.

Kriegel, H.: Hybrid simulation of the Enceladus plasma interaction and comparison with Cassini magnetometer data, Master's thesis, Technische Universität Braunschweig, 2009.

Kriegel, H., Simon, S., Wiehle, S., Kleindienst, G., Motschmann, U., Glassmeier, K.-H., Saur, J., Khurana, K., and Dougherty, M.: Hybrid simulations of the Enceladus plasma interaction and comparison with MAG data, American Geophysical Union, Fall Meeting, Abstract P23B-1386, 2008.

Ledvina, S. A., Luhmann, J. G., Brecht, S. H., and Cravens, T. E.: Titan's induced magnetosphere, Adv. Space Res., 33, 20922102, 2004.

Luhmann, J. G.: Titan's ion exosphere wake: A natural ion mass spectrometer?, J. Geophys. Res., 101, 29387-29393, 1996.
Ma, Y.-J., Nagy, A. F., Cravens, T. E., Sokolov, I. V., Clark, J., and Hansen, K. C.: 3-D global MHD prediction for the first close flyby of Titan by Cassini, Geophys. Res. Lett., 31, 1-4, 2004.

Ma, Y.-J., Nagy, A. F., Cravens, T. E., Sokolov, I. V., Hansen, K. C., Wahlund, J.-E., Crary, F. J., Coates, A. J., and Dougherty, M. K.: Comparisons between MHD model calculations and observations of Cassini flybys of Titan, J. Geophys. Res., 111, A05207, doi:10.1029/2005JA011481, 2006.

Ma, Y.-J., Nagy, A. F., Toth, G., Cravens, T. E., Russell, C. T., Gombosi, T. I., Wahlund, J.-E., Crary, F. J., Coates, A. J., Bertucci, C. L., and Neubauer, F. M.: 3D global multi-species Hall-MHD simulation of the Cassini T9 flyby, Geophys. Res. Lett., 34, 24, doi:10.1029/2007GL031627, 2007.

Masters, A., Achilleos, N., Dougherty, M. K., Slavin, J. A., Hospodarsky, G. B., Arridge, C. S., and Coates, A. J.: An empirical model of Saturn's bow shock: Cassini observations of shock location and shape, J. Geophys. Rese. (Space Physics), 113, 10210, doi:10.1029/2008JA013276, 2008.

Modolo, R. and Chanteur, G. M.: A global hybrid model for Titan's interaction with the Kronian plasma: Application to the Cassini Ta flyby, J. Geophys. Res. (Space Physics), 113, 1317, doi:10.1029/2007JA012453, 2008.

Modolo, R., Chanteur, G. M., Wahlund, J.-E., Canu, P., Kurth, W. S., Gurnett, D., Matthews, A. P., and Bertucci, C.: Plasma environment in the wake of Titan from hybrid simulation: A case study, Geophys. Res. Lett., 34, L24S07, doi:10.1029/2007GL030489, 2007.

Motschmann, U. and Kührt, E.: Interaction of the solar wind with weak obstacles: Hybrid simulations for weakly active comets and for Mars, Space Sci. Rev., 122, 197-208, doi:10.1007/s11214-006-6218-2, 2006.

Neubauer, F. M., Backes, H., Dougherty, M. K., Wennmacher, A., Russell, C. T., Coates, A., Young, D., Achilleos, N., Andre, N., Arridge, C. S., Bertucci, C., Jones, G. H., Khurana, K. K., Knetter, T., Law, A., Lewis, G. R., and Saur, J.: Titan's near magnetotail from magnetic field and plasma observations and modelling: Cassini flybys TA, TB and T3, J. Geophys. Res., 111, A10220(115), doi:10.1029/2006JA011676, 2006.

Neubauer, F. M., Gurnett, D. A., Scudder, J. D., and Hartle, R. E.: Titan's magnetospheric interaction, in Saturn, edited by T. Gehrels and M. S. Matthews, pp. 760-787, University of Arizona Press, Tucson, Arizona, 1984.

Omidi, N., Quest, K. B., and Winske, D.: Low Mach number parallel and quasi-parallel shocks, J. Geophys. Res., 95, 2071720730, 1990.

Roussos, E., Müller, J., Simon, S., Bößwetter, A., Motschmann, U., Krupp, N., Fränz, M., Woch, J., Khurana, K. K., and Dougherty, M. K.: Plasma and fields in the wake of Rhea: 3-D hybrid simulation and comparison with Cassini data, Ann. Geophys., 26, 619-637, 2008, http://www.ann-geophys.net/26/619/2008/.

Sauer, K., Bogdanov, A., and Baumgartel, K.: Evidence of an ion composition boundary (protonopause) in bi-ion fluid simulations of solar wind mass loading, Geophys. Res. Lett., 21, 2255-2258, 1994.

Simon, S.: Titan's highly variable plasma environment: A 3D hybrid simulation study, $\mathrm{PhD}$ thesis, Technische Universität Braunschweig, 2007.

Simon, S., Bagdonat, T., Motschmann, U., and Glassmeier, K.-H.: 
Plasma environment of magnetized asteroids: a 3-D hybrid simulation study, Ann. Geophys., 24, 407-414, 2006a, http://www.ann-geophys.net/24/407/2006/.

Simon, S., Bößwetter, A., Bagdonat, T., Motschmann, U., and Glassmeier, K.-H.: Plasma environment of Titan: a 3-D hybrid simulation study, Ann. Geophys., 24, 1113-1135, 2006b, http://www.ann-geophys.net/24/1113/2006/.

Simon, S., Boesswetter, A., Bagdonat, T., and Motschmann, U.: Physics of the Ion Composition Boundary: a comparative 3-D hybrid simulation study of Mars and Titan, Ann. Geophys., 25, 99-115, 2007a, http://www.ann-geophys.net/25/99/2007/.

Simon, S., Boesswetter, A., Bagdonat, T., Motschmann, U., and Schuele, J.: Three-dimensional multispecies hybrid simulation of Titan's highly variable plasma environment, Ann. Geophys., 25, 117-144, 2007b, http://www.ann-geophys.net/25/117/2007/.

Simon, S., Kleindienst, G., Boesswetter, A., Bagdonat, T., Motschmann, U., Glassmeier, K.-H., Schuele, J., Bertucci, C., and Dougherty, M. K.: Hybrid simulation of Titan's magnetic field signature during the Cassini T9 flyby, Geophys. Res. Lett., 34, L24S08, doi:10.1029/2007GL029967, 2007c.

Simon, S., Motschmann, U., and Glassmeier, K.-H.: Influence of non-stationary electromagnetic field conditions on ion pick-up at Titan: 3-D multispecies hybrid simulations, Ann. Geophys., 26, 599-617, 2008, http://www.ann-geophys.net/26/599/2008/.
Simon, S., Motschmann, U., Kleindienst, G., Saur, J., Bertucci, C. L., Dougherty, M. K., Arridge, C. S., and Coates, A. J.: Titan's plasma environment during a magnetosheath excursion: Realtime scenarios for Cassini's T32 flyby from a hybrid simulation, Ann. Geophys., 27, 669-685, 2009a, http://www.ann-geophys.net/27/669/2009/.

Simon, S., Saur, J., Neubauer, F., Motschmann, U., and Dougherty, M.: Plasma wake of Tethys: Hybrid simulations versus Cassini MAG data, Geophys. Res. Lett., 36, L04108, doi:10.1029/2008GL036943, 2009b.

Simon, S., Motschmann, U., Kleindienst, G., Glassmeier, K.-H., Bertucci, C., and Dougherty, M. K.: Titan's magnetic field signature during the Cassini T34 flyby: Comparison between hybrid simulations and MAG data, Geophys. Res. Lett., 35, L04107, doi:10.1029/2007GL033056, 2008.

Snowden, D., Winglee, R., Bertucci, C., and Dougherty, M.: Three-dimensional multifluid simulation of the plasma interaction at Titan, J. Geophys. Res. (Space Physics), 112, 12221, doi:10.1029/2007JA012393, 2007. 